\title{
Mortes-Frontières ou "Beljouissance"? Amélie Nothomb, écrivain belge
}

\section{Claire NODOT-KAUFMAN}

De tous les pays oùj'ai vécu, la Belgique est celui que j'ai le moins compris. C'est peut-être cela, être de quelque part: ne pas voir de quoi il s'agit. (Amélie Nothomb, Bibliographie 226)

$\mathrm{J}$

ouissant depuis ses débuts fracassants en 1992 d'un immense succès populaire aussi bien en France qu'à l'étranger, Amélie Nothomb a immédiatement pris sa place parmi le corpus des auteurs français, ce qui lui a valu entre autres d'être étudiée comme sujet de bac, titre de reconnaissance s'il en faut dans la littérature française. L'auteur vit à Paris et participe régulièrement à la rentrée littéraire parisienne, mais la classer comme auteur français serait néanmoins un jugement hâtif et injustifié.

Car, ne serait-ce que d'un point de vue officiel, Nothomb est Belge, et peut-être plus Belge que d'autres, car sa famille oeuvra activement à la construction de l'État belge en 1830 et à l'élection du premier roi des Belges, Léopold Ier. Pourtant, la classer comme auteur belge est également problématique. D'une part parce que la littérature belge est elle-même l'objet d'une question ouverte, et d'autre part parce que l'auteur n'a finalement connu son pays qu'à l'âge de 17 ans, confrontation qu'elle vit 
d'ailleurs comme un véritable choc, ainsi que le souligne la citation en exergue. Née au Japon et bilingue, Nothomb se revendique d'ailleurs régulièrement comme japonaise, exilée, ou apatride. Mais pour cette littérature dite belge, ou « lettres belges de langue française » (Balises 29) comme le souhaiterait Marc Quaghebeur, l'un des plus grands défenseurs d'une spécificité littéraire belge, ces revendications sont presque un lieu commun.

Cet article se propose de rechercher et d'analyser dans l'œuvre de Nothomb les occurrences de la Belgique et d'un sentiment ou d'un style belge. Qu'est-ce qui fait de Nothomb un auteur éminemment belge ? Mon étude s'articulera autour de trois points au sein de son œuvre, à savoir l'image de non lieu ou d'absence ; le rapport de l'auteur à la langue comme définition de son identité ; et ce que je nommerais, par rapport à l'idée de belgitude, comme une « beljouissance ", soit un rapport ludique par rapport à une identité belge. Je me propose de démontrer que son œuvre est effectivement empreinte de ce sentiment belge, qui se situe entre deux eaux, entre les Mortes-Frontières (lieu de l'un de ses romans, Mercure, évoquant l'idée de fragmentation, de creux et d'absence) et le jardin des délices (en référence au tableau de Jérôme Bosch, un peintre qu'elle mentionne par ailleurs dans Métaphysique des tubes, lieu de plaisir, et chez elle, lieu de jouissance de la langue et de l'identité).

Qu'est-ce qu'être Belge pour Nothomb qui n'a, après tout, connu son pays que sur le tard? L'auteur se situe en effet dans une configuration très particulière. Comme je l'ai indiqué en introduction, Nothomb est née au Japon, à Kobé, en 1967, où son père avait été envoyé en mission diplomatique en tant qu'ambassadeur de Belgique. Nothomb père s'inscrit ainsi dans la longue lignée familiale qui fait de sa famille un des piliers de son pays. Les Nothomb appartiennent en effet à l'aristocratie belge. En 1830, Jean-Baptiste Nothomb, le premier diplomate de la famille, participe à l'indépendance de la Belgique et à l'élection du roi Léopold. Ministre, il publie une Histoire de la Révolution de 1830. La famille compte quelques célébrités, notamment Pierre Nothomb, écrivain et parlementaire dans les années 30 mais également fervent nationaliste belge, et un second diplomate, en la personne de Patrick Nothomb, le père d'Amélie. Nothomb naît donc dans une famille intimement associée à l'Histoire de la Belgique. Elle se retrouve par conséquent dans une situation quelque peu paradoxale : ne connaissant pas la Belgique, elle appartient à une famille dont la mission est de la représenter et l'incarner à l'étranger.

Car la Belgique, pour elle, est un non-lieu, une abstraction. Ainsi, lorsque sa mère lui apprend que les obligations de son père vont les 
conduire à quitter le Japon, et qu'il est de son devoir d'obéir à la Belgique , Nothomb répond : «Elle est loin la Belgique » (Métaphysique 123). Et de fait, la Belgique reste toujours pour elle ce qui est loin. C'est d'ailleurs de manière très révélatrice lors de son deuxième séjour au Japon, cette fois en tant qu'adulte, qu'elle éprouve pour la première fois la nostalgie de la Belgique : « Au bout du fil, le gros accent du terroir m'émut comme jamais » (Stupeurs 38). Nothomb est Belge de loin. Le terme belge est en outre problématique, car elle ne connaît longtemps d'autre Belges que sa propre famille : « Les Belges étaient limités à trois : mon frère André, ma sœur Juliette et moi. Il n'y avait pas d'autres enfants de notre nationalité » (Sabotage 17). Elle reprend cette idée pour la renforcer dans Biographie de la faim afin de décrire son parcours scolaire. Elle est tout d'abord le seul «pissenlit belge » (45), détonnant au milieu des enfants nippons parfaitement disciplinés. Puis, elle en fait également l'expérience au Lycée français de New York: «Il n'y avait pas de Belges. J'ai remarqué ce curieux phénomène dans le monde entier : j'étais toujours la seule Belge de la classe » (111). Etre belge, c'est donc dès lors se distinguer, avoir un caractère unique. Etre belge est une curiosité, idée que l'on développera par la suite. Au mieux, si elle doit définir dans son enfance ce qu'est un Belge, elle peut tout au plus formuler qu'est belge ce qui n'est pas japonais : " Il y avait beaucoup de différences sur la terre : les Japonais et les Belges (je croyais que tous les Blancs étaient belges, sauf moi qui me tenais pour japonaise) » (Métaphysique 82). Or Nothomb, enfant, se croit et se dit Japonaise.

Mais elle n'aura pas la chance de rester ni d'être Japonaise puisque sa famille va constamment se déplacer. Et de fait, les pays s'enchaînent : après le Japon, les Nothomb sont envoyés en Chine, puis à New York, au Bangladesh, et en Birmanie. Ce n'est qu'à l'âge de 17 ans que Nothomb met enfin le pied à Bruxelles pour y mener des études de lettres. La découverte de ce pays est loin de représenter une reconnaissance, mais révèle plutôt en elle un certain malaise. Nothomb ne comprend pas le pays de ses ancêtres, elle ne voit pas " de quoi il s'agit » (Biographie 226). Déracinée, incapable aussi bien de s'insérer dans le pays qu'elle aurait voulu se choisir (le Japon) et le pays auquel elle appartient officiellement (la Belgique), Nothomb reste exilée, apatride. Quand elle demande à son père lors de leur séjour en Chine quand elle rentrera à la " maison », soit pour elle le Japon, celui-ci lui répond : "Jamais » (Biographie 85). Ce terme-là, Nothomb l'adopte pour en faire sa véritable patrie : elle sera jamaisienne. 
Jamais était le pays que j'habitais. C'était un pays sans retour. Je ne l'aimais pas. Le Japon était mon pays, celui que j'avais choisi, mais lui ne m'avait pas élue. Jamais m'avait été désignée : j'étais ressortissante de l'Etat de Jamais.

[...]

Les jamaisiens sont de grands bâtisseurs d'amours, d'amitiés, d'écritures, et autres édifices déchirants qui contiennent déjà leur ruine, mais ils sont incapables de construire une maison, une demeure, ou même quoi que ce soit qui ressemble à un logis stable et habitable. Rien, pourtant, ne leur paraît aussi digne de convoitise qu'un tas de pierres qui serait leur domicile. Une fatalité leur dérobe cette terre promise dès qu'ils croient en avoir la clé. (Biographie 85-86).

Ce pays de « Jamais », elle semble ne l'avoir plus quitté dans la vie réelle ou dans sa fiction. Ses romans se situent de manière révélatrice en plein cœur de ce non-lieu, de cette absence, de ce Jamais qu'elle a élu pour résidence. Ses intrigues ont souvent pour cadre un décor minimal, dans des endroits liminaux ou marginaux : l'aéroport de Cosmétique de l'ennemi, les toilettes de Stupeurs et tremblements, la maison isolée à la campagne des Catilinaires, le vide futuriste de l'ouvrage de sciencefiction que constitue Péplum, ou encore l'île coupée du monde et baptisée Mortes-Frontières de Mercure. Mortes-Frontières, tel est lieu d'élection de Nothomb. La marge et le vide semblent lui convenir plus que tout autre. C'est ainsi le cas dans Stupeurs et tremblements où elle fait part de son étrange obsession pour la fenêtre de l'entreprise japonaise où elle effectue un stage plus que catastrophique. La seule chose qui lui permette de tenir, exprime-t-elle à plusieurs reprises, c'est de s'imaginer se jeter dans le vide : «je passais des heures debout, le front collé au verre, à jouer à tomber dans le vide. Je voyais mon corps tomber, je me pénétrais de cette chute jusqu'au vertige » (Stupeur 140).

Mais cet attachement à la marge, ou à l'excentricité, ce sentiment lancinant d'apatride, semblent justement une donnée constituante commune aux écrivains belges. Les lettres belges de langue française seraient, selon Quaghebeur, une « littérature du non-lieu et de l'en-soi, un éther qui se déroule en dehors du réel bien divers » (Ardus 43). La Belgique ne devient-elle pas d'ailleurs chez Nothomb l'incarnation même de ce non-lieu, elle qui consacre tant de pages à ses expériences au Japon, Chine et autres pays, mais n'accorde que deux pages et demie, un tout petit chapitre, à son premier contact avec Bruxelles et son pays ? Un 
silence se fait de sa part sur la Belgique, silence aussi pesant que celui que les Belges eux-mêmes semblent attacher à leur pays, silence face aux nombreux traumas historiques jalonnant leur histoire : holocauste congolais, corruption des fonctionnaires, collaboration de la seconde guerre mondiale, question royale et question des régionalismes. $\mathrm{La}$ Belgique, pour Antoon van der Braembussche, est « a loss of memory, a loss of identity, an absence, a silence. A slip of the tongue ? » (35). Elle est également pour Quaghebeur un « manque à être " (Frickx 21), pour Hubert Juin un "pays de marge ", et enfin pour François Perrin « une nation introuvable » (Frickx 27). Quaghebeur, dans Balises pour l'histoire des lettres belges, souligne comment le texte considéré comme fondateur des lettres belges, les aventures de Thyl (Ulenspiegel) par Charles de Coster, se déroule " au pays de Flandres et ailleurs ", " comme si dès l'origine, notre littérature se fixait un horizon aux antipodes des littératures nationales » (36, je mets en italique). Et de rappeler également que Michaux, autre écrivain d'origine belge notable, avait éprouvé la nécessité de « se déplacer constamment et n'appartenir jamais » (149, je mets en italique).

Jamais, Ailleurs, telles sont donc les frontières des lettres belges de langue française. Ce sentiment de déracinement et d'étrangeté, de malaise et d'incompréhension, les auteurs belges semblent l'avoir en partage. Et il est troublant de retrouver ces mêmes traits chez Nothomb, pour autant unique qu'ait été son chemin de vie. Cette phrase de Jean Muno pourrait être la sienne au mot pour mot, jusque dans sa pointe finale :

Je me suis toujours senti très belge. C'est-à-dire double, triple, situé à un carrefour excentrique, marginal de naissance, provincial et international, bâtard d'ici et de nulle part, toujours sur une frontière, gêné aux entournures, complexé de partout [...]. En somme, bourrelé de contradictions, bizarre, bilingue, bicéphale et peut-être même biscornu. (cité par Michel Otten « Identité nationale, identité régionales», 73, je mets en italique).

Cette citation montre par ailleurs la relation étrange et étrangère par rapport à la langue que semblent cultiver les écrivains belges de langue française, et qui est commun à Nothomb. Du fait des obligations de ses parents, Nothomb ne peut s'identifier ni s'attacher à une patrie, un pays. Cela n'a pas de sens, et après la déchirure impossible qu'elle a subie en quittant le Japon, s'attacher à un pays tiendrait du pur autosabotage. Dès lors, elle réalise que pour définir son identité, il ne lui reste qu'un choix : la langue. Dans un entretien rapporté par Laureline 
Ammanieux dans son étude sur Nothomb, l'auteur tient les propos suivants : « Très vite, $\mathrm{j}$ 'ai compris que ma seule identité était la langue, puisque c'était la seule chose que je ne perdais pas tous les trois ans " (104). Son pays réside dans une pure construction linguistique. Mais de quelle langue s'agit-il au juste?

Nothomb était dans son enfance parfaitement bilingue entre le français et le japonais. On retrouve donc chez elle un rapport à la langue marqué par la diglossie, une surconscience linguistique, pour reprendre l'expression de Lise Gauvin sur les auteurs francophones, qui lui fait aborder le français comme un perpétuel territoire étranger. Dans Métaphysique des tubes, Nothomb souligne combien les premiers mots qu'elle choisit de prononcer font l'objet d'une mûre réflexion linguistique : «Chaussure? Non, ce n'est pas le plus important; on peut marcher sans. Papier ? Oui, mais c'est aussi nécessaire que crayon. [...] Harmonica. Ça sonne bien, mais est-ce vraiment indispensable ? Lunette ? Non, c'est rigolo, mais ça ne sert à rien. Xylophone ?... » (39). L'économie des mots se met immédiatement en place, ainsi que leur sonorité, évoquant déjà la " lalangue » lacanienne, sur laquelle Janet Thormann dans son étude sur "The Ethical Subject of The God of Small Things » note qu'elle matérialise " desire in letters, in materialized sounds, sensuous images, and the representation of things. Unconscious jouissance infuses the detached signifiers of lalangue, the isolated units of sound that are apparently meaninglesss conglomerations of syllables " (300).

La jouissance ne cesse dès lors d'accompagner le développement du langage chez Nothomb. Elle s'affirme également très tôt sensible aux différentes langues. Son frère et sa sœur suivant des cours à l'école américaine, elle se positionne pour le choix et la défense d'une langue qui lui soit propre. Puisqu'elle navigue aisément entre français et japonais, elle amalgame les deux langues en s'inventant son propre langage : le franponais, une langue jubilatoire, encore une fois proche de lalangue, que Nothomb elle-même qualifie de « délectable " (Biographie 47). Janet Thormann fait sensiblement la même observation sur The God of Small Things, quand elle souligne que

Language for the children is literal and oral; what they read is transformed into sounds, and what they hear has the material substance of letters. Their world is an idiosyncratic shared speech, and they produce their world and escape adult surveillance with an uncanny facility of talking backwards, or they bypass language altogether, communicating intuitively in a kind of unspoken nondifferentiation. (301, je mets en italique) 
En inventant le franponais, Nothomb se constitue bien son propre monde, et mélange intimement deux langues qui n'ont pour elle aucune différentiation : "Pour moi, il n'y avait pas des langues, mais une seule et grande langue dont on pouvait choisir les variantes japonaises ou françaises, au gré de sa fantaisie » (Métaphysique 49). Nous observons également dans sa description du franponais, la matérialité que prennent pour elle les sons :

Comment ne pas avoir faim du franponais? Ces mots aux syllabes bien détachées les unes des autres, aux sonorités nettes, c'étaient des sushis, des bouchées pralinées, des tablettes de chocolat dont chaque carré verbal se découpait facilement, c'étaient des gâteaux pour le thé de cérémonie, dont les emballages individuels permettaient le bonheur du déshabillage et la différenciation des saveurs. (47)

On voit ici très nettement comment Nothomb associe la langue à un plaisir oral, mais également comment elle parvient à manipuler la langue pour parvenir à réconcilier chez elle des extrêmes ou/et des identités multiples. La langue devient le lieu d'exploration des frontières, mais non plus mortes, comme suggéré en première partie. La frontière se fait ici vivante et jubilatoire. Quaghebeur note justement comment dans les lettres belges « la langue comble, par des tours surprenants, le manque à être qui signe la Belgique »(Frickx 21).

$\mathrm{Qu}$ 'en est-il de la question linguistique qui hante la Belgique ? Estelle ou non présente chez Nothomb ? Au cours de ses voyages, Nothomb rencontre bien en effet la diversité linguistique de son pays maternel. $\mathrm{Si}$ elle affirme que pendant longtemps il n'y eut pas d'autres Belges qu'elle et sa famille, il faudrait préciser Belge francophone. Elle fait mention dans Le Sabotage amoureux de « deux exquises petites Flamandes » (18), mais ces dernières montrant peu de velléités guerrières, Nothomb n'eut aucun véritable contact avec elles : "nous ne pûmes rien en tirer » (18). Elle reprend cet épisode de manière elliptique dans Biographie de la faim. "Révélation de la complexité nationale : je rencontrai des Belges qui ne parlaient pas français. Décidément, le monde était bien curieux » (82). La rencontre avec la troisième Belgique, celle des germanophones, a lieu à New York lorsque débarque Inge, sa jeune fille au pair : « Elle arrivait de la Belgique germanophone " (104); « elle débarquait de son village belge et n'en revenait pas de ce qu'elle voyait » (107). Inge est très certainement bilingue puisqu'il n'est fait nulle mention de son accent ou d'un 
apprentissage de l'allemand par Nothomb. Inge représente un autre élément du puzzle linguistique belge. Les derniers éléments se rencontrent dans l'épisode du Bangladesh où sœur Marie-Paule tient une léproserie : « La Belge en question était une espèce de militaire déguisée en religieuse qui s'appelait Marie-Paule » (182). Lui sont finalement adjointes deux Flamandes, sœur Lies et sœur Leen ", parlant un patois incompréhensible : «Leur langage évoquait les tremblements d'un couvercle de casserole dans laquelle des patates seraient en train de bouillir " (196). Que l'on compare cette métaphore gastronomique à celle du franponais, et l'on réalise le peu d'indulgence que Nothomb développe pour ses compatriotes non francophones.

Cette cohabitation linguistique ratée se retrouve dans Antéchrista, le seul roman de Nothomb situé en Belgique, où Blanche, Belge francophone et vraisemblablement bruxelloise, est fascinée par Christa, venue des cantons allemands de l'Est. La fascination devient rapidement cauchemar lorsque la jeune allemande vient habiter chez Blanche et vampirise petit à petit sa chambre, sa famille et tout son univers. Une des situations les plus intolérables pour Blanche consiste notamment à devoir supporter les goûts musicaux de Christa qui se passionne pour le rock allemand : «Elle mit à plein tube un morceau qui était délicatement intitulé : So schrecklich. "On ne saurait mieux dire », pensai-je » (Antéchrista 57). Affreuse cohabitation?

Enfin, il y a le rapport avec les autres francophones, non Belges ceux là, que sont les français, ég alement découverts lors du séjour chinois. Loin de positionner sa langue par rapport à la France, maison mère de la langue et référence absolue, Nothomb fait leur rencontre avec amusement, les qualifiant de "pittoresques » (Sabotage 18). Elle ne comprend pas leur demande de « parler en belge » (18), et les nomme « une peuplade qui parlait presque la même langue que la nôtre et dont elle s'était accaparé la dénomination. Leur pays s'appelait la France, était loin d'ici et possédait l'école » (Biographie 81). La France n'est pas pour elle le pays frontalier et rival habituel : il est, comme la Belgique, loin et peu concret.

La diglossie de Nothomb la rend certainement plus sensible à la langue : le mot ne va jamais de soi, il est toujours à réinventer ou plutôt à réinterpréter. Michel David souligne très justement dans son style la recherche du « prénom néologique ou encyclopédique, du mot rare, de l'ellipse, de l'oxymoron ou de l'hyperbole " (14). Mais, je voudrais mentionner également la manipulation perpétuelle chez elle du mot et du langage qui pousse ce dernier dans ses retranchements. Nothomb va jusqu'au bout du mot. Ainsi dans Antéchrista, le titre du roman est un 
exemple de cette manipulation. Le personnage qui s'oppose à l'héroïne du roman se nomme Christa, un nom tout à fait typique en Allemagne, mais que Blanche (la seule belge fictionnelle de l'œuvre de Nothomb !) trouve extraordinaire. Lorsque Christa se révèle le pur poison qu'elle est dans la vie de Blanche, cette dernière finit par la surnommer " Antéchrista » (70), poussant jusqu'au bout l'analogie Christa/Christ et Antéchrista/ Antéchrist. Elaborant plus loin la métaphore, elle se rend dans la ville de Christa, nommée Malmédy : « je m'aperçus qu'il n'y avait pas d'accent aigu sur 1'E de Malmédy, contrairement à la prononciation de mes parents et moi. Christa avait toujours dit Malmedy et non Malmédy : nous avions donc eu tort d'y voir une prononciation allemande » (116-117). Malmédy devient donc " mal me dit » et Antéchrista vient d'un lieu consacré au Mal. Nothomb privilégie également les jeux de mots et détournements d'expressions : son capitaine de Mercure se nomme Omer Loncours, car un capitaine se doit d'être « au long cours » $(\mathrm{O} /$ eau - eau=mer) ; le poids des personnages des Combustibles est évalué en livres (romans et non unité de mesure) car de leur volonté à brûler les livres pour se réchauffer dépend leur survie (17); de même, toujours dans Les Combustibles, le professeur remercie son étudiant pour « le tuyau » qui consiste à justement se réchauffer aux tuyaux (13). Il y a donc chez elle un usage récurrent du calembour. Le rapport de Nothomb à la langue transparaît comme extrêmement ludique.

S'il n'y a pas de langue belge en soi, l'écriture de Nothomb s'inscrit néanmoins dans un style belge. Lorsque Marcel Moreau veut expliquer ce que sa langue trahit de ses racines belges, il explique : «Mon entrée spasmodique, quasi vomitive en écriture, les ébranlements de syntaxe et les dislocations de pensée qui s'ensuivent, je les dois à la Belgique, non à elle en tant que telle, mais à elle mal reçue ou mal vécue par moi » (Andrianne, Écrire en Belgique 149). La Belgique n'apparaît pas ici comme une donnée concrète, tangible, mais comme une reconstruction linguistique entre malaise et jouissance. Le rapport de Nothomb à l'écriture aussi bien qu'à la Belgique est similaire. Elle appartient sans conteste à ce groupe d'écrivains ainsi définis par Marc Quaghebeur :

faisons semblant d'écrire en français, mais, en fait, minons cette façon de dire qui ne correspond pas à ce que nous voulons dire. Faisons-lui opérer, sous l'apparence du plus beau français qui soit, des décalages pouvant aller jusqu'à l'envers de ce que les mots veulent dire au sens usuel. Cela fait aussi partie d'un certain esprit belge, de Maeterlinck aux surréalistes. Ce type d'humour 
étrange qui nous caractérise surprend souvent les tiers, ce sens de l'autodérision parfois hallucinant -il peut aller jusqu'au masochisme- va de pair avec un sens de l'affirmation qui passe aussi par un sens de la dissimulation ... (Entre aventures 43).

Autodérision poussée au masochisme, décalage et inversion, affirmation et dissimulation font bien partie de la panoplie de Nothomb. Ne racontet-elle pas sa vie en affirmant que tout est vrai, pour soudain laisser entendre au détour d'une page qu'elle ment tout le temps (Métaphysique, 115)? Elle manie également cette autodérision masochiste dans les clins d'œil à elle-même qu'elle multiplie dans ses œuvres fictionnelles : « les contes élisabéthains de lady Amelia Nothumb " (Mercure, 134); son propre meurtre par son personnage dans Robert des noms propres («Elle prit le fusil qui ne la quittait pas (...) et tira sur la tempe d'Amélie », 189-190); et enfin le bizarre Péplum où une romancière du nom de A.N. est enlevée par un scientifique du futur pour avoir mis à jour son complot d'avoir volontairement détruit Pompéi. C'est pourtant paradoxalement dans ce roman de science-fiction pure que Nothomb revendique son étiquette d'écrivain belge. En effet, alors que Celsius (son kidnappeur) se moque de ses prétentions à vouloir savoir si son œuvre a survécu et traversé les siècles, il la classifie en ces termes : "Squelette d'écrivain belge du vingtième siècle » (77), ajoutant ces mots encore plus remarquables : « une curiosité $»$.

Nothomb bête curieuse de la littérature belge, ou la littérature belge elle-même une curiosité ? Est-ce à dire que le fait d'être écrivain belge est en soi une curiosité ? La position de Nothomb semble rejoindre celle de Javeau, l'inventeur du terme « belgitude », qui tint les propos suivants : «Tout ce qui est belge me sera toujours forcément étranger. Ou mieux : étrange » (Andrianne, Ecrire en Belgique 142). L'étrangeté d'être Belge a poursuivi Nothomb au cours de son enfance alors qu'elle erre de pays en pays et reste -elle et sa famille- unique. Première de sa classe au lycée français de New York et toujours la seule Belge, elle provoque l'admiration, la curiosité et une certaine incrédulité chez ses professeurs : " Vous êtes sûre que vous êtes belge ?» (Biographie 112). Etre belge demeure une " terrible vérité » (112). Etrange aux autres, étrangère en tous pays, Nothomb demeure également étrange et étrangère pour elle-même. Et de fait, je rappellerai encore la citation de Nothomb utilisée en exergue de l'introduction : «De tous les pays où j'ai vécu, la Belgique est celui que j'ai le moins compris » (Biographie 226). Cette incompréhension, loin pourtant d'être démotivante, loin de constituer des « mortes-frontières » 
freinant toute énergie créatrice, me semble au contraire essentielle au développement de l'écrivain. A priori, la Belgique pourrait sembler absente de son œuvre mais l'ellipse dissimule souvent chez Nothomb un événement fondamental. Et de fait, à y regarder de plus près, la Belgique est indirectement évoquée comme associée à trois moments absolument déterminants de son existence.

La Belgique étant absente de son enfance et de son adolescence de manière directe, Nothomb ne connaît dans un premier temps son pays que par des artéfacts. Quand la Belgique est représentée dans son enfance ou dans son œuvre, c'est sous formes d'objets et de codes culturels. Il y a par exemple la lecture: Tintin est le livre que ses parents veulent lui voir lire, la sacro-sainte Bible belge. Mais ce qui parvient à toucher Nothomb est du domaine de la nourriture. Nolens volens, le pays d'origine devient pays maternel lorsqu'il s'associe chez elle à la nourriture et au plaisir oral.

L'acte fondateur a lieu dans Métaphysique lorsque la grand-mère paternelle fait le voyage depuis la Belgique afin de rencontrer sa dernière petite-fille. Ce qui motive le voyage est que ladite petite-fille, Nothomb donc, semble sortie de la phase autistique dans laquelle elle est restée depuis sa naissance et manifeste bruyamment et continuellement sa colère. Pour apaiser le bébé, la grand-mère, assurant ainsi le passage de témoin générationnel et identitaire, lui fait découvrir le chocolat, " du chocolat blanc de Belgique » (29). C'est la première fois que les mots « Belgique » et " chocolat " sont prononcés devant l'enfant. Ils y resteront définitivement associés puisque c'est du moment qu'elle goûte le chocolat que Nothomb date la naissance conjointe du plaisir et de la conscience chez elle : «C'est moi ! C'est moi qui vis ! C'est moi qui parle ! [...] Le plaisir est une merveille, qui m'apprend que je suis moi » (30-31).

La même scène est répétée en Chine lorsque Nothomb découvre cette fois les spéculoos que ses parents lui cachent. La volupté est cette fois encore intense, si intense qu'elle se précipite devant un miroir pour se regarder : « je voulais me voir en état de plaisir. Ce qu'il y avait sur mon visage, c'était le goût du spéculoos » (Biographie 89). Ce « spéculoos spéculaire " (David 113) renforce donc le lien entre Belgique et plaisir oral. Lors de son séjour aux Etats-Unis, Nothomb n'aura donc aucune difficulté à expliquer à ses interlocuteurs américains ce qu'est la Belgique. Lorsqu'on la confond avec une Bulgare, elle trouve la parfaite définition : " Je me lançai dans une explication sur mes origines véritables : je venais de Belgique, c'était le pays qui avait inventé le spéculoos, le chocolat y était meilleur qu'ailleurs » (Biographie 141, je mets en italique). Notons que pour la première fois dans le cours de son autobiographie, Nothomb 
se définit enfin comme Belge, et non plus comme Japonaise comme elle l'a longtemps cru. Elle y affirme même " venir de Belgique ", un comble si l'on considère qu'elle n'y a encore jamais mis les pieds. Mais la grâce du chocolat et du spéculoos, de la découverte du plaisir à travers quelque chose de spécifiquement belge, lui fait choisir cette identité.

En même temps que la Belgique, elle rencontre donc la jouissance (et vice et versa), ou ce que $j$ 'ai choisi de nommer la « beljouissance » par rapport au terme « belgitude » forgé par Javeau. La belgitude est à l'origine formulée sur le modèle de la négritude, et signifie « not only the fact of being Belgian, but the fact of feeling at home in Belgium " (Braembussche 35). Or Nothomb repousse absolument ce terme qu'elle juge impropre, en ajoutant: «Ce que je n'aime pas dans la belgitude, c'est la notion de souffrance : tout mot qui se termine par-tude a l'air d'impliquer quelque chose de douloureux. Or moi, franchement, je suis très contente d'être belge " (Bainbrigge 182, je mets en italique). Je propose donc le terme " beljouissance " pour définir le rapport de Nothomb à son identité belge, découverte à travers le plaisir oral de la nourriture, et réaffirmée à travers le plaisir de l'écriture.

En effet le troisième événement fondamental lié à la Belgique dans la vie de Nothomb sera la venue à l'écriture. Comme je l'ai signalé à plusieurs reprises, Nothomb ne consacre qu'un très court chapitre à sa découverte in situ de la Belgique, mais elle y fait cet aveu qui mérite qu'on s'y attache : "C'est peut-être cela, être de quelque part : ne pas voir de quoi il s'agit. Sans doute est-ce pour cela que j'y commençai à écrire. Ne pas comprendre est un sacré ferment pour l'écriture. Mes romans mettaient en forme une incompréhension qui croissait » (Biographie 226). Si Nothomb commence à écrire pour des raisons psychologiques (la fin de son anorexie), c'est néanmoins le passage par la Belgique qui lui permet de réaliser l'acte, un acte qu'elle qualifie dans de nombreux entretiens et romans comme un acte de pure jouissance : «Si un écrivain ne jouit pas, alors il doit s'arrêter à l'instant. Ecrire sans jouir, c'est immoral » (Hygiène de l'assassin, 85). Confrontée à son pays d'origine, un pays qu'elle ne comprend pas, Nothomb éprouve le besoin d'écrire, de combler le " manque à être " évoqué à plusieurs reprises précédemment. La Belgique devient la page blanche idéale, la tabula rasa qui appelle à l'écriture. Mais en même temps, on pourrait dire que l'incompréhension de Nothomb face à son pays d'origine, ce sentiment de vide, a simplement répondu à quelque chose d'intrinsèque en elle, et que la Belgique a révélé.

On a déjà vu chez elle ce sentiment profond de liminalité, de marginalité, d'excentricité (au sens, éloigné du centre). Or la Belgique 
répond à tout cela en elle. Il est donc naturel que le malaise qu'elle éprouve au début face à ce pays, en faisant écho à son propre malaise/mal-être intérieur, lui permette de se libérer. La Belgique est pleinement ce pays à la conjonction des mortes frontières qui habitent l'écrivain et de sa formidable envie de jouir. L'auteur se retrouve à écrire symboliquement -sinon physiquement- en Belgique, soit le domaine du liminaire, du paradoxal. Sur sa conception de l'écriture, elle révèle que le fait d'écrire la « maintient à la frontière entre cohérence et folie pure, entre ce qui a du sens et ce qui n'en a pas, entre quelque chose et rien du tout. Cette frontière je la sens physiquement » (David 177). Cette frontière, ce territoire de l'écriture est bien la Belgique, « le pays le plus imaginaire du monde » (Jean de Decker cité par Andrianne, Écrire en Belgique 139), dont on se sauve justement par l'imagination. Le monde baroque, fantasmatique et insolite de Nothomb est proprement belge.

"Aucun Belge n'est semblable à moi ", proclame fièrement Nothomb dans Stupeurs et tremblements, 71. Mais qu'est-ce qu'un Belge ? C'est cette indétermination même qui résout l'auteur belge, quel qu'il soit, à n'assumer d'autres frontières que celles de l'écriture et de la langue, celles qu'il s'est choisi et qu'il s'approprie. Si Nothomb ne se représente pas dans ses romans autobiographiques comme Belge, elle n'a par contre aucune difficulté à se représenter comme écrivain belge, aussi bien dans ses romans fictionnels que dans ses entretiens, où elle revendique sans conteste son appartenance à cet étrange et composite microcosme littéraire, parfois honteux, parfois belge malgré lui, souvent méconnu. Mais son univers parle de la Belgique, implique « quelque chose comme la Belgique » (Hubert Juin, cité par Frickx, 26) mieux que si elle situait explicitement tout son univers dans le monde belge. C'est également bien un jardin des délices à la Jérôme Bosch, univers composite et grotesque, un paradis qui peut dérouter, voire rebuter plus d'un, mais où l'auteur se refuse à tout interdit. Un motif récurrent dans ses romans autobiographiques la rattache même au symbole de la Belgique par excellence, le Manneken Pis. Nothomb se décrit urinant debout dans $L e$ sabotage_amoureux 1, assise en classe dans Biographie de la faim, sans parler de sa fonction de dame pipi dans Stupeurs et tremblements. Or ces scènes ou cette fonction, elle l'assume avec un grand éclat de rire, voire une fierté non dissimulée dans le premier roman. Ces scènes évoquent irrésistiblement le petit bonhomme joufflu, devenu icône de Bruxelles, et auquel fait pendant la Jeanneke Pis, gamine insolente, dont Nothomb recommande tout particulièrement la découverte (Zumkir 26). En évoquant le souvenir de ces deux icônes bruxelloises, l'auteur fait un portrait 
humoristique d'elle-même en représentante de son pays : Amélie Pis, ou l'écrivain belge par excellence.

University of Wisconsin

\section{Notes}

1. J'annonçai comme un musicien préciserait 'allegro ma non troppo' avant un morceau :-Debout sans les mains. (...) Je m'en allai à pas lents. Mon visage n'affichait rien. Je délirais d'orgueil. (96)

\section{Ouvrages cités}

Amanieux, Laureline. Amélie Nothomb, l'éternelle affamée. Paris: Albin Michel, 2005.

Andrianne, René. Ecrire en Belgique. Bruxelles: Labor, 1983.

"Conscience linguistique et conscience politique." Colloque de Louvain-la-Neuve, 20 avril 1982: Ecriture française et identifications culturelles en Belgique. Éd. Pierre Watté. Louvainla-Neuve: Ciaco, 1984. 11-24.

Ardus, Fabienne. Discours de l'altérité en Belgique francophone contemporaine: Claire Lejeune, Caroline Lamarche, Nicole Malinconi et Amélie Nothomb. Lafayette: U of Southwestern Louisiana, 1999.

Bainbrigge, Susan et Jeanette den Tooder, ed. Amélie Nothomb: Authorship, Identity and Narrative Practice. NY: Peter Lang, 2001. Braembussche, Antoon van de. "The Silence of Belgium: Taboo and Trauma in Belgian Memory." Yale French Studies, 102 (2002): 34-52. David, Michel. Amélie Nothomb : le symptôme graphomane. Paris: L'Harmattan, 2006.

Frickx, Robert. "Littérature belge de langue française ou littérature française de Belgique?" Acte du colloque international de Soleure, juin 1993: L'identité culturelle de la Belgique et de la Suisse francophones. Ed. Paul Gorceix. Paris: Honoré Champion, 1997.21-30.

Nothomb, Amélie. Hygiène de l'assassin. Paris: Albin Michel, 1992.

-. Le Sabotage amoureux. Paris: Albin Michel, 1993.

- Les Combustibles. Paris: Albin Michel, 1994.

—. Les Catilinaires. Paris: Albin Michel, 1995.

-. Péplum. Paris: Albin Michel, 1996.

-. Mercure. Paris: Albin Michel, 1998.

-. Stupeurs et tremblements. Paris: Albin Michel, 1999. 
-. Métaphysique des tubes. Paris: Albin Michel, 2000.

-. Cosmétique de l'ennemi. Paris: Albin Michel, 2001.

-. Robert des noms propres. Paris: Albin Michel, 2002.

-. Antéchrista. Paris: Albin Michel, 2003.

-. Bibliographie de la faim. Paris: Albin Michel, 2004.

Otten, Michel. "Identité nationale, identités régionales." Colloque de Louvain-la-Neuve, 20 avril 1982: Ecriture française et identifications culturelles en Belgique. Ed. Pierre Watté. Louvainla-Neuve: Ciaco, 1984. 49-83.

Perrin, François. Histoire d'une nation introuvable. Bruxelles: Paul Legrain, 1988.

Quaghebeur, Marc. Balises pour l'histoire des lettres belges. Bruxelles : Labor, 1982.

-. Entre aventures, syllogismes et confessions. Bruxelles: Peter Lang, 2003.

Thormann, Janet. "The Ethical Subject of The God of Small Things." JPCS: Journal for the Psychoanalysis of Culture \& Society, 8.2 (2003): 299-307.

Zumkir, Michel. Amélie Nothomb de A à Z. Bruxelles: Le grand miroir, 2003.

\section{Ouvrages consultés}

Albert, Christiane, ed. Francophonie et identités culturelles. Paris: Karthala, 1999.

Craen, Piet van de. "What, if Anything, Is a Belgian?" Yale French Studies 102(2002): 24-33.

Gohard-Radenkovic, Aline, ed. Altérité et identités dans les littératures de langue française. Le français dans le monde v. 334. Paris: Clé international, 2004.

Heller, Monica. Codeswitching. Berlin: Mouton de Gruyter, 1988.

Makward, Christiane and Odile Cazenave. "The Others's Others: 'Francophone' Women and Writing." Yale French Studies 75 (1988): 190-207. 
